\title{
Detection of MicroRNA-21 Expression as a Potential Screening Biomarker for Colorectal Cancer: a Meta-analysis
}

\author{
Jian-Xin Jiang ${ }^{1,2 \&}, \mathrm{Na}_{\text {Zhang }}^{3 \&}$, Zhong-Min Liu ${ }^{1 *}$, Yan-Ying Wang ${ }^{1,4 *}$
}

\begin{abstract}
Background: Colorectal cancer (CRC) is a major cause of cancer-related death and cancer-related incidence worldwide. The potential of microRNA-21 (miR-21) as a biomarker for CRC detection has been studied in several studies. However, the results were inconsistent. Therefore, we conducted the present meta-analysis to systematically assess the diagnostic value of miR-21 for CRC. Materials and Methods: Using a random-effect model, the pooled sensitivity (SEN), specificity (SPE), positive likelihood ratio (PLR), negative likelihood ratio (NLR), and diagnostic odds ratio (DOR) were calculated to evaluate the diagnostic performance of miR-21 for CRC. A summary receiver operating characteristic (SROC) curve and an area under the curve (AUC) were also generated to assess the diagnosis accuracy of miR-21 for CRC. Q test and I2 statistics were used to assess betweenstudy heterogeneity. Publication bias was evaluated by the Deeks' funnel plot asymmetry test. Results: A total of 986 CRC patients and 702 matched healthy controls from 8 studies were involved in the meta-analysis. The pooled results for SEN, SPE, PLR, NLR, DOR, and AUC were $57 \%$ (95\% CI: 39\%-74\%), 87\% (95\% CI: 78\% 93\%), 4.4 (95\% CI: 2.4-8.0), 0.49 (95\% CI: 0.32-0.74), 9 (95\% CI: 4-22), and 0.83 (95\% CI: 0.79-0.86), respectively. Subgroup analyses further suggested that blood-based studies showed a better diagnostic accuracy compared with feces-based studies, indicating that blood may be a better matrix for miR-21 assay and CRC detection. Conclusions: Our findings suggest that miR-21 has a potential diagnostic value for CRC with a moderate level of overall diagnostic accuracy. Hence, it could be used as auxiliary means for the initial screening of CRC and avoid unnecessary colonoscopy, which is an invasive and expensive procedure.
\end{abstract}

Keywords: MicroRNA-21 - colorectal cancer - meta-analysis - diagnosis

Asian Pac J Cancer Prev, 15 (18), 7583-7588

\section{Introduction}

Colorectal cancer (CRC) is the third most common cancer and ranks second to lung cancer, with more than 1.2 million new cases being diagnosed per year worldwide (Janakiram and Rao, 2008; Jemal et al., 2011). Meanwhile, it is the fourth most common cause of death from cancer worldwide (Jemal et al., 2011). It is comforting to know that CRC is curable by surgical resection if detected early. The 5-year relative survival rate of CRC patients reaches over $93 \%$ for stage I. As for stage IV disease, this rate falls to less than $8 \%$ (O'Connell et al., 2004). Unfortunately, there are no significant symptoms of CRC at the initial stage. Thus, due to the outstanding decline of the mortality rate of CRC at an early stage, efficient diagnostic methods by which the cancer can be detected earlier are urgently needed.

To date, the gold standard for diagnosing CRC is colonoscopy, which is an invasive and expensive procedure (Kanaan et al., 2012a). But this method requires both unpleasant bowel preparation and sedation. What's more, it may lead to some medical complications. So it is not difficult to understand the reason why many patients are reluctant to receive a colonoscopy test. Therefore, the wide application of colonoscopy is hampered although it is considered reliable and credible. On the other hand, the fecal occult blood test (FOBT) is also adopted most widely as the screening test of CRC for now (Wu et al., 2012a). It is a non-invasive method through detecting fecal blood, which may leak from disrupted vessels of CRC patients. However, the low sensitivity of FOBT may result in missing a large proportion of CRC patients (Imperiale et al., 2004). As the rapid development of several diagnostic techniques, various kinds of early detection method have been proposed. These methods focus on kinds of biomarkers, including neoplasm-derived proteins, DNA and RNA markers. Their applications are also restricted because of low sensitivity. For instance, the sensitivity and specificity of carcinoembryonic antigen (CEA) screening in blood for the differentiation of CRC patients from healthy controls are $74 \%$ and $87 \%$, respectively (Fakih and Padmanabhan, 2006). The researches that Imperiale et al.

${ }^{1}$ Research Center for Translational Medicine, ${ }^{2}$ Department of Neurosurgery, ${ }^{4}$ Department of Gastroenterology, East Hospital, Tongji University School of Medicine, Shanghai, ${ }^{3}$ Key Laboratory of Food Science and Engineering of Heilongjiang Province, Harbin University of Commerce, Harbin, China ${ }^{\star}$ Equal contributors*For correspondence: yanying_wang@126.com,zhongmliu@163.com 
and Ahlquist et al. conducted showed that the sensitivity of the individual DNA markers varies from $3.2 \%$ to $25.8 \%$ while the sensitivity of the combined panel of 23 DNA markers is 52\% (Imperiale et al., 2004; Ahlquist et al., 2008). Other frequently exploited analytes, such as fecesbased messenger RNA (mRNA), were also suggested to be able to detect CRC (Wu et al., 2012a). Although many efforts have been focused on identification of novel biomarkers for CRC, compliance with them has been far from adequate. Hence, reliable, precise and noninvasive biomarkers for diagnosis and prediction of CRC are still urgently needed.

MicroRNAs (miRNAs) are a family of small and non-coding RNA molecules with 18-25 nucleotides in length. MiRNAs function as regulators of gene expression by binding to the 3' untranslated regions of target mRNAs (Koga et al., 2010a; Kanaan et al., 2012a). Recent researches have demonstrated the important role of miRNAs in the development and progression of CRC and consequently make miRNAs as potential biomarkers for CRC diagnosis. More specifically, miR-221, miR-29a and miR-92a were suggested to be abnormally expressed in CRC patients in many studies (Ng et al., 2009; Huang et al., 2010; Pu et al., 2010). Besides, several studies reported high expression levels of miR-21, miR-135 and miR-17-92 cluster in CRC patients (Monzo et al., 2008; Nagel et al., 2008; Diosdado et al., 2009; Yamamichi et al., 2009). The stability of miRNAs in blood or feces makes them more promising for the early diagnosis of CRC. Accordingly, we can reach the conclusion that miRNAs may serve as biomarkers of CRC screening.

MiR-21, as one of the most extensively studied oncogenic miRNAs, is supposed to be able to modulate the expression of multiple cancer-related genes (Meng et al., 2007; Zhu et al., 2007; Asangani et al., 2008). It's also the earliest discovered miRNA in body fluids secreted by tumor cells (Lawrie et al., 2008). Accumulating evidence showed the aberrant expression of miR-21 in various human tumors, including CRC (Xu et al., 2013; Huang et al., 2013). In addition, expression level of miR-21 was statistically significantly elevated in CRC patients and increased during tumor progression (Slaby et al., 2007; Schetter et al., 2008; Shibuya et al., 2010; Chang et al., 2011). However, the findings concerning the diagnostic accuracy of miR-21 as a biomarker for CRC failed to achieve consensus. As far as we know, there have been no meta-analysis to systematically evaluate the diagnostic accuracy of miR-21 for CRC screening currently. Therefore, a meta-analysis of all the data from the included studies is of great value. Moreover, conducting a metaanalysis can transcend the limitations of the single study, such as small sample size, thereby providing a more precise outcome. Consequently, to systematically evaluate the value of miR-21 assays in early CRC detection is exactly the aim of the present study.

\section{Materials and Methods}

Search strategy

We conducted a comprehensive literature search to identify relevant studies prior to March 29, 2014. Sources of studies included PubMed, Embase, the Cochrane Library, Web of Science and Chinese National Knowledge Infrastructure (CNKI) databases. The following search terms were adopted: ("microRNA-21" or "miRNA-21" or "miR-21") and ("colorectal cancer" or "CRC") and ("diagnosis" or "ROC curve" or "sensitivity" or "specificity"). For further relevant studies, the references of selected articles were screened manually to identify any additional relevant studies.

\section{Inclusion and exclusion criteria}

A studies was considered eligible if it met the follow inclusion criteria: (1) concerns the evaluation of miR-21 in the diagnosis of CRC; (2) the sensitivity and specificity data were presented or can be calculated; (3) matched healthy controls were included The exclusion criteria were as follows: (1) studies with duplicate data reported in other studies; (2) studies that were editorials, letters, case reports or reviews; (3) studies without complete data to allow construction of two-by-two tables.

\section{Data extraction}

All the included studies were carefully reviewed by two investigators independently, then the date from these articles such as author; publication year; country of study; ethnicity of patients; number, age and gender of patients and controls; source of control; type of specimen; test method of miRNA-21 expression; miRNAs expression change; sensitivity and specificity data were extracted. When the accurate data of sensitivity and specificity were not presented in the articles directly, we estimated the values from the receiver operator characteristic (ROC) curves manually.

\section{Quality assessment}

The methodological quality of each study that included in the diagnostic meta-analysis was assessed by two independent investigators using the Quality Assessment of Diagnostic Accuracy Studies-2 (QUADAS-2) criteria (Whiting et al., 2011). The QUADAS-2 tool used seven questions to evaluate the quality of diagnostic accuracy of the studies and each question should be answered with "yes", "no", or "unclear". A maximum possible score is 7 while one score was given for each "yes", and no scores were given for each "no" and "unclear".

\section{Statistical methods}

The statistical analyses were performed by STATA 12.0 software. The pooled results of the sensitivity (SEN), specificity (SPE), positive likelihood ratio (PLR), negative likelihood ratio (NLR), diagnostic odds ratio (DOR) with their $95 \%$ confidence intervals (CIs) were calculated using random-effect model. Among these parameters, PLR is defined as the odds of positive test results in patients with CRC, while NLR reflects the odds of positive results in those without the disease. Correspondingly, DOR, the value of which ranges from 0 to infinity, is the ratio of PLR to NLR. In general, higher DOR values indicate better discriminatory test performance (Glas et al., 2003). Simultaneously, we plot the summary receiver operator characteristic (SROC) curve by using the sensitivity and 
specificity of each included study, and the area under the SROC curve (AUC) was calculated. Besides, Q statistic and $\mathrm{I}^{2}$ test were used to assess the heterogeneity in the included studies. P value less than 0.1 for $\mathrm{Q}$ statistic and $\mathrm{I}^{2}$ more than $50 \%$ indicated the existence of significant heterogeneity (Higgins et al., 2003; Dinnes et al., 2005). Furthermore, meta-regression and subgroup analyses were conducted to detect the heterogeneity between studies. Publication bias was also evaluated using Deeks' funnel plots (Deeks et al., 2005).

\section{Results}

Included studies

The initial search returned a total of 72 manuscripts and 3 additional records were found though other sources. Among the 82 records, 4 were excluded for duplicate data. After manually screening the titles, abstracts and key words, 45 records were removed because they were editorials, letters, case reports, or studies irrelevant to our analysis. The full-text visions of the remained 33 literatures were carefully read and 25 records that did not correspond to the inclusion criteria were excluded: 21 articles were not diagnostic test studies, and no available data for the other 4 articles. Thus, 8 high-quality studies (Koga et al., 2010b; Kanaan et al., 2012b; Kuriyama et al., 2012; Wu et al., 2012b; Liu et al., 2013; Luo et al., 2013; Toiyama et al., 2013; Zanutto et al., 2014) were eligible for our systematic review and meta-analysis.

\section{Baseline characteristics}

The main characteristics of the included articles are listed in Table 1 by order of publication year, which ranges from 2010 to 2014 . The 8 studies included in the present meta-analysis contains 968 CRC patients who have been confirmed using histopathology and 702 matched healthy controls. Overall, 5 studies were conducted in Asian populations and 3 in Caucasian populations. All the studies detected the expression level of miR-21 through quantitative real-time polymerase chain reaction (qRTPCR) assay, while five of them tested it in blood samples and three in feces. Seven of the eight studies described a significant increase in the expression level of miR-21 in CRC patients compared to healthy controls. All in all, the eligible studies were of very good quality with moderately high QUADAS-2 scores.

\section{Diagnostic accuracy of miR-21 in CRC}

Forest plots of data from the included studies and mean sensitivity and specificity with corresponding heterogeneity are shown in Figure 1. There appeared to be significant heterogeneity between studies in sensitivity and specificity $\left(\mathrm{I}^{2}=96.90 \%\right.$ and $\mathrm{I}^{2}=91.01 \%$, respectively). Hence, the random-effect model was adopted for the metaanalysis. The pooled results for diagnostic accuracy are listed in Table 2 . The overall sensitivity and specificity were 0.57 (95\%CI: 0.39-0.74) and 0.87 (95\%CI: 0.78$0.93)$, respectively. Other overall parameters were also calculated and presented in the table: pooled PLR was 4.4 (95\%CI: 2.4-8.0), NLR was 0.49 (95\%CI: 0.32-0.74), and DOR was 9 (95\%CI: 4-22). Moreover, we generated the SROC curve as shown in Figure 2 while the area under the curve (AUC) was 0.83 and its $95 \% \mathrm{CI}$ was $0.79-0.86$. The results suggested that miR-21 had a good level of overall accuracy.

\section{Subgroup analyses and meta-regression}

To access the heterogeneity between studies, subgroup analyses based on ethnicity (Asian or Caucasian) and sample type (blood-based or feces-based) were conducted. As a result, studies based on Asian turned out to have a similar sensitivity and specificity compared to the pooled estimates. In respect to the subgroup analysis which was aimed to figure out the difference between blood-based and feces-based samples, the former showed a better diagnostic accuracy with sensitivity increased to 0.70 .

Table 1. Main Characteristics of 6 Studies Included in Meta-Analysis

\begin{tabular}{|c|c|c|c|c|c|c|c|c|c|c|c|}
\hline \multirow[t]{2}{*}{ First author } & \multirow[t]{2}{*}{ Year } & \multirow[t]{2}{*}{ Country } & \multirow[t]{2}{*}{ Ethnicity } & \multicolumn{2}{|c|}{ Sample size } & \multicolumn{2}{|c|}{ Mean age (yr) } & \multirow{2}{*}{ Specimen } & \multirow[t]{2}{*}{ Sensitivity } & \multirow[t]{2}{*}{ Specificity } & \multirow[t]{2}{*}{ QUADAS-2 } \\
\hline & & & & Cases & Controls & Cases & Controls & & & & \\
\hline Koga et al & 2010 & Japan & Asian & 197 & 119 & 63 & 60 & Feces & $14.70 \%$ & $91.60 \%$ & 3 \\
\hline Kanaan et al & 2012 & USA & Caucasian & 50 & 50 & 60 & 61 & Plasma & $81.00 \%$ & $94.00 \%$ & 6 \\
\hline Kuriyama et al & 2012 & Japan & Asian & 138 & 126 & NA & NA & Feces & $39.00 \%$ & $97.60 \%$ & 7 \\
\hline Wu et al & 2012 & China & Asian & 88 & 101 & 67.2 & 60.5 & Feces & $55.70 \%$ & $73.30 \%$ & 4 \\
\hline Liu et al & 2013 & China & Asian & 200 & 80 & NA & NA & Serum & $65.00 \%$ & $85.00 \%$ & 3 \\
\hline Luo et al & 2013 & Germany & Caucasian & 80 & 144 & 68 & 62.5 & Plasma & $53.00 \%$ & $82.00 \%$ & 6 \\
\hline Toiyama et al & 2013 & Japan & Asian & 186 & 53 & 67.5 & 64 & Serum & $82.80 \%$ & $90.60 \%$ & 4 \\
\hline Zanutto et al & 2014 & Italy & Caucasian & 29 & 29 & NA & NA & Plasma & $58.00 \%$ & $58.00 \%$ & 5 \\
\hline
\end{tabular}

*NA, not available; QUADAS, quality assessment of diagnostic accuracy studies

Table 2. Summary Estimates of Diagnostic Criteria and the 95\% Confidence Intervals

\begin{tabular}{lllr}
\hline Analysis & \multicolumn{1}{c}{ Overall } & \multicolumn{1}{c}{ Asian } & Blood-based \\
\hline Sensitivity $(95 \%$ CI) & $0.57(0.39-0.74)$ & $0.51(0.28-0.74)$ & $0.70(0.55-0.81)$ \\
Specificity (95\% CI) & $0.87(0.78-0.93)$ & $0.90(0.80-0.95)$ & $0.85(0.75-0.92)$ \\
Positive LR (95\% CI) & $4.4(2.4-8.0)$ & $5.0(2.4-10.3)$ & $4.7(2.4-9.3)$ \\
Negative LR (95\% CI) & $0.49(0.32-0.74)$ & $0.54(0.34-0.88)$ & $0.36(0.22-0.59)$ \\
DOR (95\% CI) & $9.0(4-22)$ & $9.0(3-26)$ & $13.0(4-42)$ \\
AUC & $0.83(0.79-0.86)$ & $0.85(0.82-0.88)$ & $0.86(0.82-0.88)$ \\
\hline
\end{tabular}

CI, confidence interval; LR, likelihood ratio; DOR, diagnostic odds ratio; AUC, area under the curve 

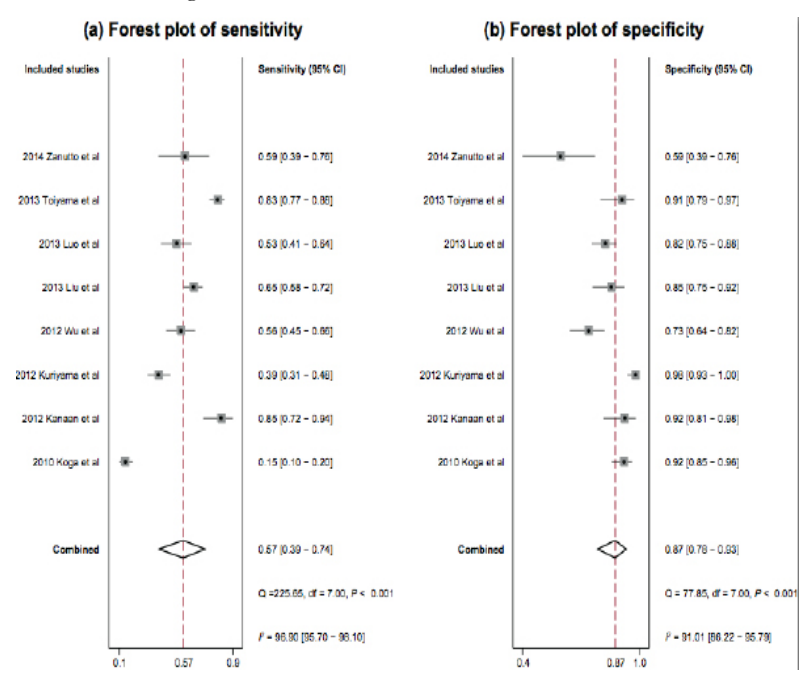

Figure 1. Forest Plot Showing Study-Specific (Rightaxis) and Mean Sensitivity and Specificity with Corresponding Heterogeneity Statistics

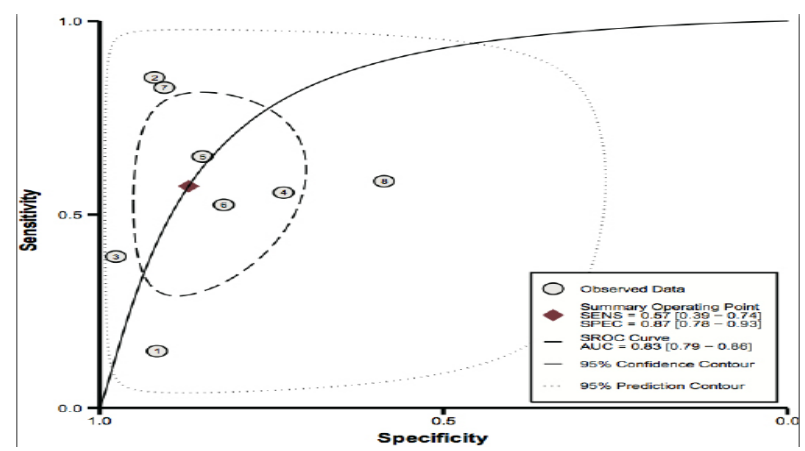

Figure 2. Summary ROC Curve with Confidence and Prediction Regions Around Mean Operating Sensitivity and Specificity Point

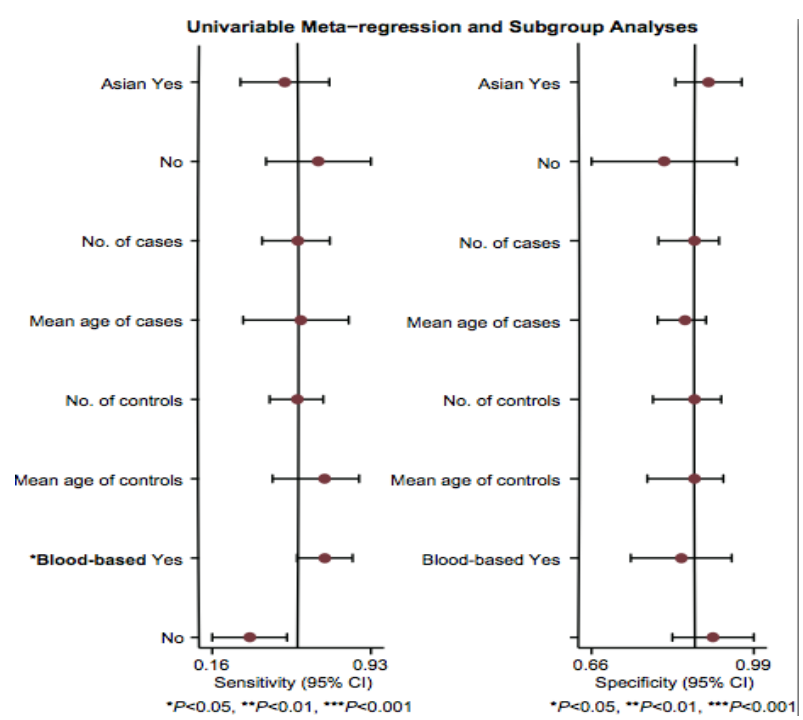

Figure 3. Forest Plots of Multivariable MetaRegression and Subgroup Analyses for Sensitivity and Specificity

For further confirmation, we also conducted the metaregression. The results that presented in Figure 3 indicated that sample type had a significant influence on sensitivity with a $\mathrm{P}$ value less than 0.05 . This in turn approved the subgroup analysis above.

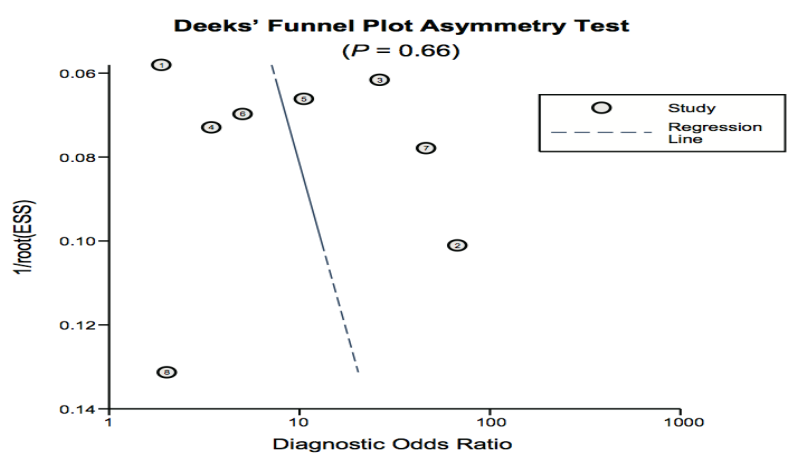

Figure 4. Linear Regression Test of Deeks' Funnel Plot Asymmetry

\section{Sensitivity analysis and publication bias}

In this meta-analysis, we also conduct the sensitivity analysis. According to the diagrams, each included study had only minimal influence on the overall estimates and no outlier studies were found. Hence, the randomeffect bivariate model was robust for the calculation of the pooled estimates. Finally, the Deeks' funnel plot asymmetry test was used to access the publication bias. As shown in Figure 4, with a P value of 0.66 for the slope coefficient, the funnel plots are almost symmetric, which also suggests a low likelihood of publication bias.

\section{Discussion}

Recently, extensive efforts have been taken to identify the diagnostic value of miR-21, which is located in chromosome 17q23.1 and is the site of translocation breakpoints or amplifications in kinds of cancers. During the past decade, numerous studies have approved the relationship between miR-21 and tumorigenesis. And there have been numerous meta-analyses about miR-21 for cancer detection. In the meta-analysis of Wang et al., the pooled sensitivity and specificity of miR-21 in cancer diagnosis were $75.7 \%$ (95\%CI: $67.5 \%-82.65 \%$ ) and $79.3 \%$ (95\%CI: $74.2 \%-83.5 \%$ ), respectively (Wang et al., 2014). Chan et al. have also demonstrated that miR-21 was over-expressed in tissues of $92 \%$ patients with gastric cancer (Chan et al., 2008). Concretely, Zeng et al. have conducted a meta-analysis which showed that the pooled sensitivity and specificity by using miR-21 to detect gastric cancer were 0.665 (95\%CI: 0.550-0.763) and 0.831 (95\%CI: $0.694-0.915)$ respectively (Zeng et al., 2013). Moreover, Li et al. have obtained the result that the detection of miR-21 expression yielded $78.80 \%$ sensitivity and $100.00 \%$ specificity in the diagnosis of lung cancer at optimum conditions ( $\mathrm{Li}$ et al., 2011). To the best of our knowledge, the current study is the first mate-analysis focusing on the overall diagnostic accuracy of miR-21 for CRC screening.

Our meta-analysis revealed that utilizing miR-21 as a biomarker for the detection of CRC yielded an overall sensitivity of $57 \%$ and an overall specificity of $87 \%$. The $57 \%$ sensitivity indicated that miR-21 was only able to identify a little more than half of the CRC patients, which meant that miR-21 might not be qualified to diagnose CRC. In addition, the pooled PLR was only 4.4, which also approved that miR-21 couldn't confirm CRC. Whereas, 
the specificity was relatively high, implying the usefulness of miR-21 as a screening biomarker. Moreover, the DOR, with higher values indicating better discriminatory test performance, was only 9 in the pooled results. The area under the curve of the SROC (AUC), which is an indicator of test performance and accounts for the trade-off between sensitivity and specificity, was 0.83 in the present metaanalysis, reflecting a relatively high level of diagnostic accuracy. Taken together, it is encouraging that miR-21 showed a relative moderate diagnostic value. Hence, it could be used as auxiliary means for the initial screening of CRC and avoid the unnecessary colonoscopy, which is an invasive and expensive procedure.

Heterogeneity is an important consideration while interpreting the results for the meta-analysis and therefore the subgroup analyses were conducted. However, there's no big difference between different ethnicities. On the other hand, the blood-based samples showed a significantly higher accuracy than the feces-based samples. A potential explanation for this outcome is the different stability of miR-21 in different samples. Besides, different laboratories may take different sample size or mean age, which also contributes to sources of heterogeneity. Correspondingly, a meta-regression was performed and as a result, neither of the factors is the source of heterogeneity. Apart from the analyses we have done, there are other factors causing heterogeneity; for instance, the threshold effect. Due to individual difference, different cut-offs or thresholds may be used in different studies, thus threshold effect arises. What's more, mir-21 levels of CRC patients in different stages may be different and this may also influence our results. With regard to this, further studies are still needed to obtain thorough evidences.

The present meta-analysis has several advantages. First of all, our study is the first to demonstrate the application potential of miR-21 in the early detection of CRC. Secondly, while retrieving literatures for analysis, rigorous screening process have been adopted, thus the included studies were of high quality. What's more, comprehensive methods, such as subgroup analyses and meta-regression (Figure 3), were conducted to minimize the influence of heterogeneity. In addition, there was no significant publication bias in our meta-analysis (Figure 4). It is all these merits that make the statistical power of the meta-analysis stronger.

Despite the above advantages, there are still several limitations that should be acknowledged in our study. Firstly, the basic characteristics of included studies were not coherent, which might limit the quality of this systematic review. The time of sample collection might have influences on the sensitivity and specificity, considering that miRNA levels might decrease after surgeries. What's more, in the light of the meta-regression that presented in Figure 3, the sample based on blood introduced significant heterogeneity. Furthermore, we failed to get the full text of an included article, which may also give rise to the uncertainty of the basic characteristic. Accordingly, multi-center studies with exhaustive analyses are needed to probe into heterogeneity. Secondly, despite we didn't apply any restrictions about language during our literature search, some other language publications, like Japanese and German, may not be included in this study, which may affect the pooled results. Besides, no studies conducted in African populations were included in current review. Finally, since only 8 articles were included in this meta-analysis, more studies are needed to get a definitive conclusion. As with all new techniques, the diagnostic accuracy of miR-21 is expected to improve with the development of detection technology.

In summary, although a limited sample size was used in this study, the role of miR-21 as a diagnostic biomarker for CRC has been assessed in this meta-analysis. Despite the sensitivity of miR-21 was not excellent, the specificity and AUC were relatively high, which indicated that miR21 might serve as a promising biomarker for the diagnosis of CRC. Besides, detection of miR-21 in blood or feces samples possesses the advantages of noninvasive and convenience. Nevertheless, further clinical studies are needed to confirm the diagnostic value of miR-21 for $\mathrm{CRC}$, and more studies should tend to study the diagnostic accuracy of CRC in an early stage.

\section{References}

Ahlquist DA, Sargent DJ, Loprinzi CL, et al (2008). Stool DNA and occult blood testing for screen detection of colorectal neoplasia. Ann Intern Med, 149, 441-50.

Asangani IA, Rasheed SA, Nikolova DA, et al (2008). MicroRNA-21 (miR-21) post-transcriptionally downregulates tumor suppressor Pdcd4 and stimulates invasion, intravasation and metastasis in colorectal cancer. Oncogene, 27, 2128-36.

Chan SH, Wu CW, Li AF, et al (2008). miR-21 microRNA expression in human gastric carcinomas and its clinical association. Anticancer Res, 28, 907-11.

Chang KH, Miller N, Kheirelseid EA, et al (2011). MicroRNA-21 and PDCD4 expression in colorectal cancer. Eur J Surg Oncol, 37, 597-603.

Deeks JJ, Macaskill P and Irwig L (2005). The performance of tests of publication bias and other sample size effects in systematic reviews of diagnostic test accuracy was assessed. J Clin Epidemiol, 58, 882-93.

Dinnes J, Deeks J, Kirby J, et al (2005). A methodological review of how heterogeneity has been examined in systematic reviews of diagnostic test accuracy. Health Technol Assess, 9, 1-113.

Diosdado B, van de Wiel MA, Terhaar Sive Droste JS, et al (2009). MiR-17-92 cluster is associated with 13q gain and c-myc expression during colorectal adenoma to adenocarcinoma progression. Br J Cancer, 101, 707-14.

Fakih MG and Padmanabhan A (2006). CEA monitoring in colorectal cancer. What you should know. Oncology, 20, 579-87.

Glas AS, Lijmer JG, Prins MH, et al (2003). The diagnostic odds ratio: a single indicator of test performance. J Clin Epidemiol, 56, 1129-35.

Higgins JP, Thompson SG, Deeks JJ, et al (2003). Measuring inconsistency in meta-analyses. BMJ, 327, 557-60.

Huang Q, Liu L, Liu CH, et al (2013). MicroRNA-21 regulates the invasion and metastasis in cholangiocarcinoma and may be a potential biomarker for cancer prognosis. Asian Pac J Cancer Prev, 14, 829-34.

Huang Z, Huang D, Ni S, et al (2010). Plasma microRNAs are promising novel biomarkers for early detection of colorectal cancer. Int J Cancer, 127, 118-26. 
Jian-Xin Jiang et al

Imperiale TF, Ransohoff DF, Itzkowitz SH, et al (2004). Fecal DNA versus fecal occult blood for colorectal-cancer screening in an average-risk population. $N$ Engl J Med, 351, 2704-14.

Janakiram NB, Rao CV (2008). Molecular markers and targets for colorectal cancer prevention. Acta Pharmacol Sin, 29, $1-20$.

Jemal A, Bray F, Center MM, et al (2011). Global cancer statistics. CA Cancer J Clin, 61, 69-90.

Kanaan Z, Rai SN, Eichenberger MR, et al (2012b). Plasma MiR-21: A potential diagnostic marker of colorectal cancer. Ann Surg, 256, 544-51.

Koga Y, Yasunaga M, Takahashi A, et al (2010a). MicroRNA expression profiling of exfoliated colonocytes isolated from feces for colorectal cancer screening. Cancer Prev Res, $\mathbf{3}$, 1435-42.

Koga Y, Yasunaga M, Takahashi A, et al (2010b). MicroRNA expression profiling of exfoliated colonocytes isolated from feces for colorectal cancer screening. Cancer Prev Res, $\mathbf{3}$, 1435-42.

Kuriyama S, Hamaya Y, Yamada T, et al (2012). Fecal microrna assays as a marker for colorectal cancer screening. Gastroenterology, 142, 770.

Lawrie CH, Gal S, Dunlop HM, et al (2008). Detection of elevated levels of tumour-associated microRNAs in serum of patients with diffuse large B-cell lymphoma. Br J Haematol, 141, 672-5.

Li Y, Li W, Ouyang Q, et al (2011). Detection of lung cancer with blood microRNA-21 expression levels in Chinese population. Oncol Lett, 2, 991-4.

Liu GH, Zhou ZG, Chen R, et al (2013). Serum miR-21 and miR-92a as biomarkers in the diagnosis and prognosis of colorectal cancer. Tumour Biol, 34, 2175-81.

Luo X, Stock C, Burwinkel B, et al (2013). Identification and evaluation of plasma microRNAs for early detection of colorectal cancer. PLOS ONE, 8, 62880.

Meng F, Henson R, Wehbe-Janek H, et al (2007). MicroRNA-21 regulates expression of the PTEN tumor suppressor gene in human hepatocellular cancer. Gastroenterology, 133, 647-58.

Monzo M, Navarro A, Bandres E, et al (2008). Overlapping expression of microRNAs in human embryonic colon and colorectal cancer. Cell Res, 18, 823-33.

Nagel R, le Sage C, Diosdado B, et al (2008). Regulation of the adenomatous polyposis coli gene by the miR-135 family in colorectal cancer. Cancer Res, 68, 5795-802.

$\mathrm{Ng}$ EK, Chong WW, Jin H, et al (2009). Differential expression of microRNAs in plasma of patients with colorectal cancer: a potential marker for colorectal cancer screening. Gut, 58, 1375-81.

O'Connell JB, Maggard MA and Ko CY (2004). Colon cancer survival rates with the new American Joint Committee on Cancer sixth edition staging. J Natl Cancer Inst, 96, 1420-5.

Pu XX, Huang GL, Guo HQ, et al (2010). Circulating miR-221 directly amplified from plasma is a potential diagnostic and prognostic marker of colorectal cancer and is correlated with p53 expression. J Gastroenterol Hepatol, 25, 1674-80.

Schetter AJ, Leung SY, Sohn JJ, et al (2008). MicroRNA expression profiles associated with prognosis and therapeutic outcome in colon adenocarcinoma. JAMA, 299, 425-36.

Shibuya $H$, Iinuma $H$, Shimada R, et al (2010). Clinicopathological and prognostic value of microRNA-21 and microRNA-155 in colorectal cancer. Oncology, 79, 313-20.

Slaby O, Svoboda M, Fabian P, et al (2007). Altered expression of miR-21, miR-31, miR-143 and miR-145 is related to clinicopathologic features of colorectal cancer. Oncology, 72, 397-402.
Toiyama Y, Takahashi M, Hur K, et al (2013). Serum miR-21 as a diagnostic and prognostic biomarker in colorectal cancer. J Natl Cancer Inst, 105, 849-59.

Wang Y, Gao X, Wei F, et al (2014). Diagnostic and prognostic value of circulating miR-21 for cancer: a systematic review and meta-analysis. Gene, 533, 389-97.

Whiting PF, Rutjes AW, Westwood ME, et al (2011). QUADAS-2: a revised tool for the quality assessment of diagnostic accuracy studies. Ann Intern Med, 155, 529-36.

Wu CW, Ng SSM, Dong YJ, et al (2012b). Detection of miR92a and miR-21 in stool samples as potential screening biomarkers for colorectal cancer and polyps. Gut, 61, 739-45.

$\mathrm{Xu} \mathrm{YZ}, \mathrm{Xi} \mathrm{QH}, \mathrm{Ge} \mathrm{WL}$, et al (2013). Identification of serum microRNA-2 1 as a biomarker for early detection and prognosis in human epithelial ovarian cancer. Asian Pac J Cancer Prev, 14, 1057-60.

Yamamichi N, Shimomura R, Inada K, et al (2009). Locked nucleic acid in situ hybridization analysis of miR-21 expression during colorectal cancer development. Clin Cancer Res, 15, 4009-16.

Zanutto S, Pizzamiglio S, Ghilotti M, et al (2014). Circulating miR-378 in plasma: a reliable, haemolysis-independent biomarker for colorectal cancer. Br J Cancer, 110, 1001-7.

Zeng Z, Wang J, Zhao L, et al (2013). Potential role of microRNA-21 in the diagnosis of gastric cancer: a metaanalysis. PLoS One, 8, 73278 .

Zhu S, Si ML, Wu H, et al (2007). MicroRNA-21 targets the tumor suppressor gene tropomyosin 1 (TPM1). J Biol Chem, 282, 14328-36. 